\title{
JUURNAL_RU
}

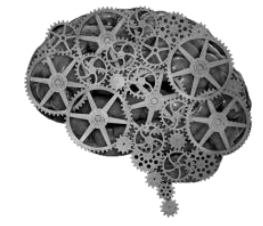

COMPANY GROUP "INTELLEKT"

Распарин В.Н. МАОУ «Гимназия №1 Октябрьского района г. Саратова»

Саратов, Россия

doi: 10.18411/lj2016-3-95

\section{Об одном исследовательском проекте}

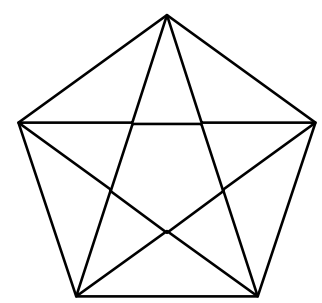

Если нарисовать правильный пятиугольник - пентагон (от греч. pentagỖnon), и в нем провести все диагонали, то получим пятиконечную звезду, называемую пентаграммой или пентаклем. Название пентаграмма происходит от греческого слова pentagrammon (pente - пять, grammon - линия) и означает правильный пятиугольник, на сторонах которого построены равнобедренные треугольники одинаковой высоты.

Из «Начал» Евклида пришла задача о делении отрезка в крайнем и среднем отношении. Суть задачи в следующем.

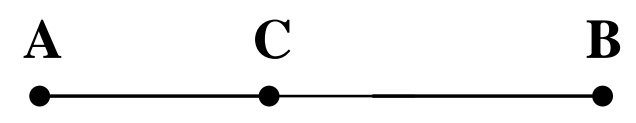

Из «Начал» Евклида пришла задача о делении отрезка в крайнем и среднем отношении. Суть задачи в следующем.

Разделим отрезок $\mathrm{AB}$ точкой $\mathrm{C}$ в таком отношении, чтобы меньшая часть его относилась к большей, как большая часть отрезка относится к отрезку $\mathrm{AB}$, то есть $\frac{A C}{C B}=\frac{C B}{A B}$

или, что то же самое: 


$$
\frac{A B}{C B}=\frac{C B}{A C} .
$$

Обозначим каждое из двух последних отношений через $X$. Тогда с учетом того, $A B=A C+C B$,

перепишем последнюю пропорцию в виде

$$
x=\frac{A C+C B}{C B}=\frac{A C}{C B}+1=\frac{1}{\frac{C B}{A C}}+1=\frac{1}{x}+1 .
$$

Откуда следует уравнение

$$
x^{2}-x-1=0
$$

Ясно, что решением этого уравнения должно быть положительное число, следовательно, решением задачи о делении отрезка в крайнем и среднем отношении должно быть число

$$
\frac{1+\sqrt{5}}{2}
$$

Это число называется золотой пропорцией, а уравнение

$$
x^{2}-x-1=0
$$

или уравнение

$$
x^{2}=x+1
$$

уравнением золотой пропорции. Приближенное значение числа

$$
\frac{1+\sqrt{5}}{2}
$$

равно 1,6180339887498948482045868343656381177203...

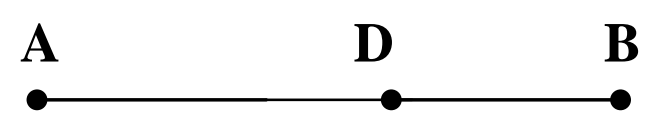

Заметим, что на отрезке $\mathrm{AB}$ существует еще одна точка, D, которая также делит его золотым сечением

$$
\frac{A B}{A D}=\frac{A D}{D B}=\frac{1+\sqrt{5}}{2} .
$$

Точки пересечения диагоналей в пентагоне всегда являются точками «божественной пропорции». При этом образуется новый пентагон, в котором можно провести диагонали, их пересечение даст еще один пентагон и так до бесконечности. Таким образом, соотношение линейных сегментов в 
пятиконечной звезде всегда равно золотой пропорции, которая обозначается буквой $\Phi($ phi)в честь выдающегося греческого скульптора Фидия (Phidias), который широко использовал ее в своих скульптурах.

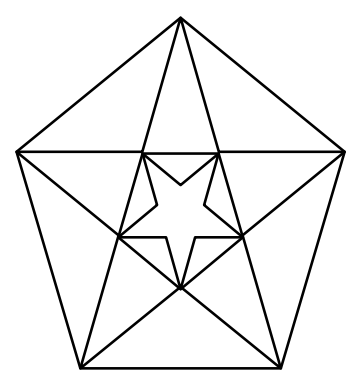

Считается, что именно Леонардо да Винчи ввел термин «золотое сечение» (aurea sectio) и использовал пропорции этого сечения во многих своих знаменитых произведениях ( в частности в «Тайной вечере» и «Моне Лизе»). Это удивительное число стало эстетическим каноном древнегреческого искусства и искусства Возрождения. Однако существует мнение, что термин восходит к Клавдию Птолемею - великому античному астроному и географу. Но термин стал популярным и закрепился в науке благодаря Леонарду да Винчи, то есть он стал поистине «кодом да Винчи».

Итак, пентагон и пентакл - один из самых могущественных образов, считавшийся во многих культурах одновременно божественным и магическим.

Учитывая упомянутое выше, традиционно своим учащимся я предлагаю задачи, связанные с пентагоном, пентаклем, с золотой пропорцией.

Так в 9 классе с целью осуществления проектно-исследовательской деятельности учащимся была предложена связка (дидактическая цепочка) задач:

В тупоугольном равнобедренном треугольнике АВС ( $\mathrm{AC}$ - основание, $\angle A=36^{0}$ ) проведена биссектриса АК. Найти отношение

$$
S_{A C K}: S_{A B K} \text {. }
$$

В равнобедренном треугольнике $\mathrm{ABC}$ с основанием

проведена биссектриса АК. Найти отношении

$$
\operatorname{AC}\left(\angle B=36^{\circ}\right)
$$

$$
S_{A B K}: S_{A C K} \text {. }
$$

На гипотенузе $\mathrm{AB}$ прямоугольного треугольника $\mathrm{ABC}$, один из острых 
углов которого составляет 25\% другого, взяты такие точки $\mathrm{M}$ и $\mathrm{N}$, что треугольники $\mathrm{ACN}, \mathrm{CMN}, \mathrm{BCM}$ - равнобедренные. Найдите следующие отношения:

$$
\begin{gathered}
\frac{S_{A M C}}{S_{C M N}} ; \frac{S_{C M N}}{S_{C N B}} ; \\
S_{A M C}: S_{C M N}: S_{C N B} .
\end{gathered}
$$

Геометрической основой предложенных задач явились так называемые «звездные» треугольники - треугольники составляющие пентаграмму.
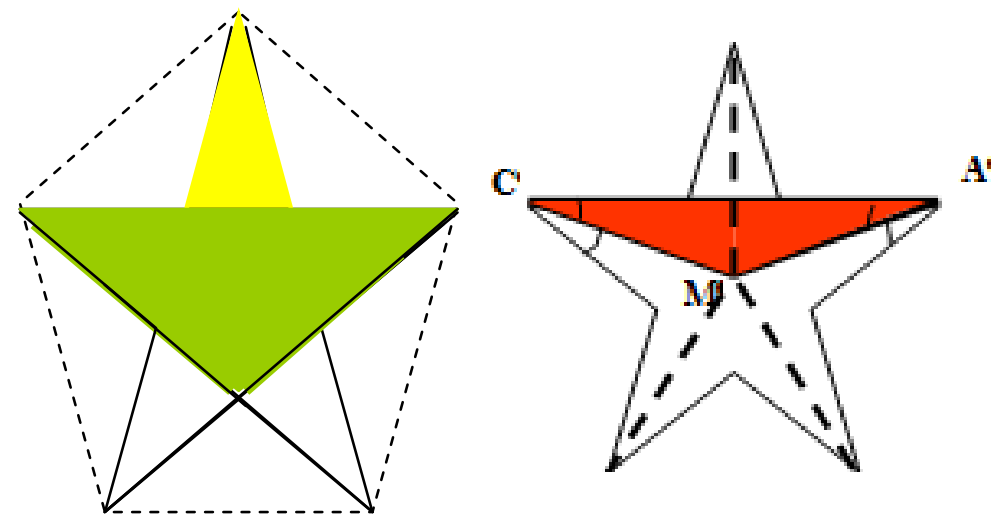

треугольники поистине «звездные»! Углы в этих треугольниках $18^{0}, 36^{0}, 54^{0}, 72^{0}, 90^{0}, 108^{0}$ - составляют (кстати!) арифметическую прогрессию.

Решение задач основано на применении метода площадей, тесным образом (что очень важно) связано с тригонометрией и алгеброй. Показать, что полученные отношения равны

$$
\frac{1+\sqrt{5}}{2}
$$

то есть являются золотой пропорцией - это только одна из целей предложенных исследовательских заданий. Другой же целью является вычисление точного значения $\sin 18^{\circ}$ разными способами, как результата решения конкретной математической модели возникшей геометрической ситуации, чтобы потом использовать полученные данные в решении задач на нахождение элементов треугольника.

Также полезно иметь ввиду, что получив значение $\sin 18^{0}$, можно 
построить, к примеру, трисекцию углов $54^{0}, 108^{\circ}$, да и сам пентагон.

Рассмотрим решения предложенных задач.

Задача 1. В тупоугольном равнобедренном треугольнике $\mathrm{ABC}$ ( $\mathrm{AC}$ основание, $\angle A=36^{0}$ ) проведена биссектриса АК. Найти отношение $S_{A C K}: S_{A B K}$.

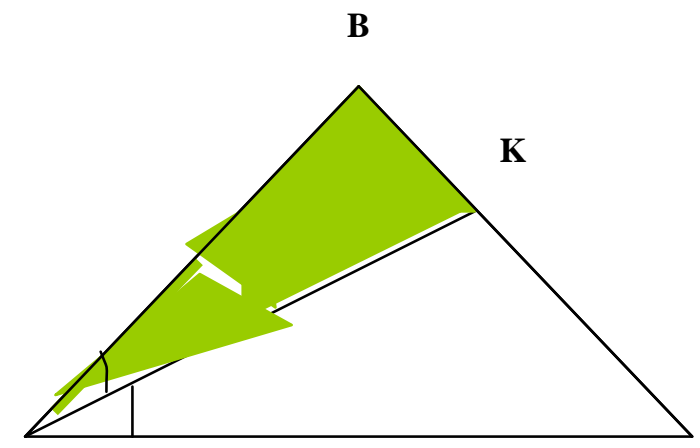

A

C

Решение.

Очевидно, что $\angle B=108^{\circ}, \angle A=36^{\circ}, \angle C=36^{\circ}$.

$$
\frac{S_{A C K}}{S_{A B K}}=\frac{C K}{B K}=\frac{A C}{A B}=2 \cdot \frac{\frac{1}{2} A C}{A B}=2 \cos 36^{\circ}=2 \sin 54^{\circ} .
$$

Итак, $\cos 36^{\circ}=\sin 54^{\circ}$.

Для ответа на вопрос нужно найти значение $\cos 36^{\circ}$ или $\sin 54^{\circ} \cdot$ Пусть $18^{\circ}=\alpha$, тогда равенство $\cos 36^{\circ}=\sin 54^{\circ}$ примет вид: $\cos 2 \alpha=\sin 3 \alpha$.

Используя формулы синуса тройного угла и косинуса двойного угла, получим уравнение $4 \sin ^{3} \alpha-2 \sin ^{2} \alpha-3 \sin \alpha+1=0$. Решая его, получим корни:

$$
1, \frac{-1-\sqrt{5}}{4}, \frac{-1+\sqrt{5}}{4} \text {. }
$$

Два первых значения $\sin \alpha$ не удовлетворяют условию задачи ввиду того, что $0<\sin 18^{\circ}\langle 1$. Поэтому

$$
\sin 18^{0}=\frac{\sqrt{5}-1}{4}
$$

Опять воспользовавшись формулой косинуса двойного угла, получим 
значение выражения $2 \cos 36^{\circ}$. Оно равно

$$
\frac{1+\sqrt{5}}{2}
$$

следовательно,

$$
S_{A C K}: S_{A B K}=\frac{\sqrt{5}+1}{2},
$$

а это золотая пропорция!

Задача 2. В равнобедренном треугольнике АВС с основанием АС ( $\angle B=36^{0}$ ) проведена биссектриса АК. Найти отношение $S_{A B K}: S_{A C K}$.

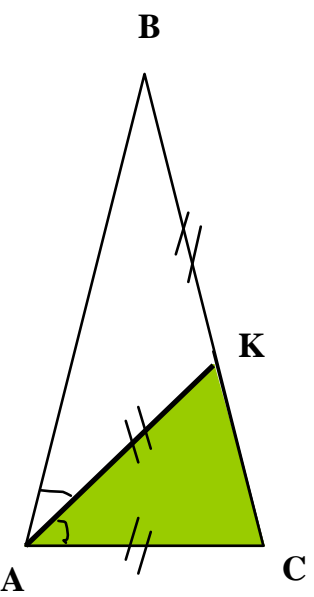

Решение.

Из условия задачи следует, что $\angle A C K=72^{\circ}, \angle C A K=36^{\circ}$.

$$
\frac{S_{A B K}}{S_{A C K}}=\frac{B K}{C K}=\frac{A B}{A C}=\frac{1}{\frac{A C}{A B}}=\frac{1}{2 \cdot \frac{\frac{1}{2} A C}{A B}}=\frac{1}{2 \sin 18^{0}}=\frac{1}{2 \cos 72^{\circ}} .
$$

Для ответа на вопрос нужно найти значение $\sin 18^{\circ}$.

Если иметь в виду то, что $18^{\circ}=\alpha$, тогда равенство $\cos 72^{\circ}=\sin 18^{\circ}$ примет вид: $\cos 4 \alpha=\sin \alpha$. Применив формулы косинуса двойного угла и синуса двойного угла к выражению $\cos 4 \alpha$, получим уравнение $1-8 \sin ^{2} \alpha+8 \sin ^{4} \alpha=\sin \alpha$, то есть $8 \sin ^{4} \alpha-8 \sin ^{2} \alpha-\sin \alpha+1=0$. будет.

Приведем его к виду $(\sin \alpha-1) \cdot\left(8 \sin ^{3} \alpha+8 \sin ^{2} \alpha-1\right)=0$.

В силу того, что $0\left\langle\sin 18^{\circ}\langle 1\right.$, значение $\sin \alpha=1$ решением задачи не

Уравнение $8 \sin ^{3} \alpha+8 \sin ^{2} \alpha-1=0$ решим используя замену $2 \sin \alpha=t$.

Тогда уравнение $8 \sin ^{3} \alpha+8 \sin ^{2} \alpha-1=0$ будет выглядеть так: $t^{3}+2 t^{2}-1=0$. Разложив его левую часть на множители, получим:

$(t+1) \cdot\left(t^{2}+t-1\right)=0$. С учетом ограничений на переменную $t$ получим, 
что $\sin 18^{\circ}=\frac{\sqrt{5}-1}{4}, \frac{1}{2 \sin 18^{0}}=\frac{\sqrt{5}+1}{2}$. Ответ получен.

Замечание. Видимо проще значение $\sin 18^{0}$ получить из треугольника AKC.

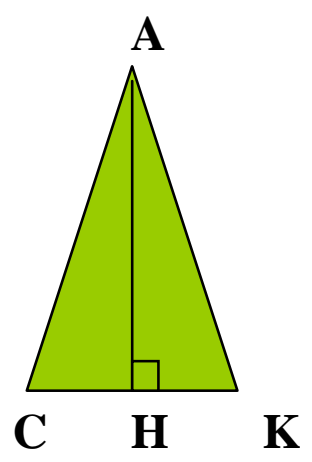

Для этого проведем высоту АН в этом треугольнике.

$\angle C A H=18^{\circ}, \angle A C H=72^{\circ}$ и $\cos 18^{\circ}=\sin 72^{\circ}$. Далее поступаем так:

$\cos 18^{\circ}=2 \sin 36^{\circ} \cos 36^{\circ}, \cos 18^{\circ}=4 \sin 18^{\circ} \cos 18^{\circ}\left(1-2 \sin ^{2} 18^{\circ}\right)$,

$1=4 \sin 18^{\circ}\left(1-2 \sin ^{2} 18^{\circ}\right), 8 \sin ^{3} 18^{0}-4 \sin 18^{\circ}+1=0$. Введя обозначение

$2 \sin 18^{0}=x$, найдем корни получившегося уравнения $x^{3}-2 x+1=0$.

Учтем, что $0<2 \sin 18^{\circ}\left\langle 2 \sin 30^{\circ}=1\right.$. И опять получим значение $\sin 18^{\circ}$.

Задача 3. На гипотенузе $\mathrm{AB}$ прямоугольного треугольника $\mathrm{ABC}$, один из острых углов которого составляет $25 \%$ другого, взяты такие точки $\mathrm{M}$ и $\mathrm{N}$, что треугольники ACN, CMN, BCM - равнобедренные. Найдите следующие

отношения: а) $\frac{S_{A M C}}{S_{C M N}}$ б) $\frac{S_{C M N}}{S_{C N B}}$ в) $S_{A M C}: S_{C M N}: S_{C N B}$.

Решение.

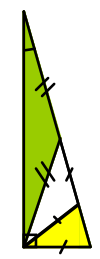

Ясно, что острые углы данного треугольника равны 180 и 720 .

Из условия задачи следует, что СМ - медиана треугольника АВС. Введем 
обозначения $B C=a, B N=b$. Тогда $M N=a, A M=a+b_{\text {и }}$ $S_{A M C}: S_{C M N}: S_{C N B}=(a+b): a: b .(*)$

Выразим $b$ через $a$, используя треугольники $\mathrm{ABC}$ и СРВ (CР - высота треугольника CNB).

$$
\text { в } \triangle \mathrm{ABC} \sin 18^{\circ}=\frac{a}{2(a+b)}, \text { в в } \triangle \mathrm{CPB} \sin 18^{\circ}=\frac{b}{2 a} .
$$

Из этих двух равенств следует, что $\frac{a}{2(a+b)}=\frac{b}{2 a}$.

Далее в результате преобразований приходим к уравнению

$$
\left(\frac{a}{b}\right)^{2}-\frac{a}{b}-1=0
$$

(а это уравнение золотой пропорции), положительным корнем которого является число

$$
\frac{\sqrt{5}+1}{2} \text {, то есть } \frac{a}{b}=\frac{\sqrt{5}+1}{2} \text {, откуда } b=\frac{2 a}{\sqrt{5}+1} .
$$

Теперь преобразуем равенство (*).

$$
\begin{gathered}
S_{A M C}: S_{C M N}: S_{C N B}=(a+b): a: b=\left(a+\frac{2 a}{\sqrt{5}+1}\right): a: \frac{2 a}{\sqrt{5}+1}= \\
=\left(1+\frac{2}{\sqrt{5}+1}\right): 1: \frac{2}{\sqrt{5}+1}=\left(1+\frac{2(\sqrt{5}-1)}{4}\right): 1: \frac{2}{\sqrt{5}+1}=\frac{\sqrt{5}+1}{2}: 1: \frac{2}{\sqrt{5}+1} .
\end{gathered}
$$

Ответ на вопрос в) получен. Ответим на вопросы а) и б).

$$
\text { a) } \frac{S_{A M C}}{S_{C M N}}=\frac{\sqrt{5}+1}{2} \text {. б) } \frac{S_{C M N}}{S_{C N B}}=\frac{1}{\frac{2}{\sqrt{5}+1}}=\frac{\sqrt{5}+1}{2} \text {. }
$$

Из решения этой задачи можно сделать выводы о том, что:

1) точка $\mathrm{M}$ и точка $\mathrm{N}$ делят соответственно отрезки $\mathrm{AN}$ и $\mathrm{BM}$ в отношении золотой пропорции;

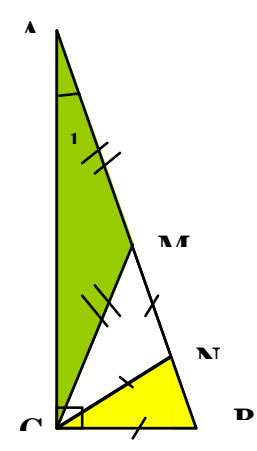


(для отрезка AN

для отрезка ВM:

$$
\frac{M N}{A M}=\frac{A M}{A N}, \frac{a}{a+b}=\frac{a+b}{2 a+b} . \Rightarrow a^{2}-a b-b^{2}=0 .
$$

$$
\left.\frac{B N}{M N}=\frac{M N}{B M}, \frac{b}{a}=\frac{a}{a+b} . \Rightarrow a^{2}-a b-b^{2}=0 .\right)
$$

2) отношения $\frac{S_{A M C}}{S_{C M N}}$ и $\frac{S_{C M N}}{S_{C N B}}$ равны золотой пропорции;

3) учитывая соотношение между величинами $a$ и $b$, полученное в ходе решения, можно теперь уже иным способом вычислить значение $\sin 18^{0}$.

$$
\left(\sin 18^{\circ}=\frac{b}{2 a}=\frac{2 a}{\sqrt{5}+1}:(2 a)=\frac{1}{\sqrt{5}+1}=\frac{\sqrt{5}-1}{4} .\right)
$$

4) зная значение $\sin 18^{0}$, можно с помощью циркуля и линейки построить трисекции углов $54^{0}, 108^{0}$, разделить прямой угол на пять равных углов, построить пентаграмму.

Важно, наконец, по окончании проекта, «помочь сделать учащимся открытие» того, что рассматриваемые треугольники являются элементами, составляющими пентаграмму.

\section{Литература:}

1. Васютинский Н.А. Золотая пропорция. М., 1990.

2. Волошинов А.В. Математика и искусство. М., 1992.

3. Воробьёв Н.Н. Числа Фибоначчи. М., 1992.

4. Ковалёв Ф.В. Золотое сечение в живописи. Киев, 1989.

5. $\quad$ Стахов А.П. Коды золотой пропорции. М., 1984.

6. Шевелёв И.Ш. Золотое сечение. М., 1990. 